\title{
The existence of symmetric positive solutions for a seconder-order difference equation with sum form boundary conditions
}

Yanping Guo', Yude $\mathrm{Ji}^{2 *}$ and Xuefei Lv²

${ }^{\text {*Correspondence: }}$
jiyude-1980@163.com
${ }^{2}$ College of Sciences, Hebei
University of Science and
Technology, Shijiazhuang, Hebei
050018, P.R. China
Full list of author information is
available at the end of the article

\begin{abstract}
In this paper, we consider the existence of positive solutions for a second-order discrete boundary value problem $\Delta(g(k-1) \Delta u(k-1))+w(k) f(k, u(k))=0$ subject to the boundary conditions: $a u(0)-b g(0) \Delta u(0)=\sum_{i=1}^{n-1} h(i) u(i)$, $a u(n)+b g(n-1) \Delta u(n-1)=\sum_{i=1}^{n-1} h(i) u(i)$, where $a, b>0, \Delta u(k)=u(k+1)-u(k)$ for $k \in\{0,1, \ldots, n-1\}, g(k)>0$ is symmetric on $\{0,1, \ldots, n-1\}, w(k)$ is symmetric on $\{0,1, \ldots, n\}, f:\{0,1, \ldots, n\} \times[0,+\infty)$ is continuous, $f(k, u)=f(n-k, u)$ for all $(k, u) \in\{0,1, \ldots, n\} \times[0,+\infty)$, and $h(i)$ is nonnegative and symmetric on $\{0,1, \ldots, n\}$. By the fixed point theorem and the Hölder inequality, we study the existence of symmetric positive solutions for the above difference equation with sum form boundary conditions.
\end{abstract}

Keywords: difference equation; sum form boundary conditions; symmetric positive solutions

\section{Introduction}

A class of boundary value problems (BVPs) with integral boundary conditions arise in thermal conduction problems, semiconductor problems, and hydrodynamic problems [1-3]. Recently, such problems have been investigated by many authors [4-10]. The equation $\left(g(t) u^{\prime}(t)\right)^{\prime}+w(t) f(t, u(t))=0,0<t<1$, describes many phenomena in the fields of gas dynamics, nuclear physics, chemically reacting systems and atomic structures [11-15]. In [10], Feng considered the following differential equation BVP with integral boundary conditions:

$$
\begin{aligned}
& \left(g(t) u^{\prime}(t)\right)^{\prime}+w(t) f(t, u(t))=0, \quad 0<t<1, \\
& a u(0)-b \lim _{t \rightarrow 0^{+}} g(t) u^{\prime}(t)=\int_{0}^{1} h(s) u(s) \mathrm{d} s, \\
& a u(1)+b \lim _{t \rightarrow 1^{-}} g(t) u^{\prime}(t)=\int_{0}^{1} h(s) u(s) \mathrm{d} s .
\end{aligned}
$$

Applying the fixed point index theorem and the Hölder inequality, the author studied the existence of symmetric positive solutions for BVP (1.1)-(1.3).

\section{空 Springer}

C2014 Guo et al.; licensee Springer. This is an Open Access article distributed under the terms of the Creative Commons Attribution License (http://creativecommons.org/licenses/by/2.0), which permits unrestricted use, distribution, and reproduction in any medium, provided the original work is properly cited. 
Motivated by the above works, we will study the following BVP with sum form boundary conditions:

$$
\begin{aligned}
& \Delta(g(k-1) \Delta u(k-1))+w(k) f(k, u(k))=0, \quad k \in\{1, \ldots, n-1\} \\
& a u(0)-b g(0) \Delta u(0)=\sum_{i=1}^{n-1} h(i) u(i) \\
& a u(n)+b g(n-1) \Delta u(n-1)=\sum_{i=1}^{n-1} h(i) u(i) .
\end{aligned}
$$

Throughout this paper, the following conditions are assumed:

$\left(\mathrm{A}_{1}\right) a, b>0, w(k)$ is symmetric on $\{0,1, \ldots, n\}$, and there exists $m>0$ such that $w(k) \geq$ $\frac{m}{n-1}$ on $\{0,1, \ldots, n\}, g(k)>0$ for $k \in\{0,1, \ldots, n\}$, and $g(k)$ is symmetric on $\{0,1, \ldots, n-$ $1\}, h$ is nonnegative, symmetric on $\{0,1, \ldots, n\}$, and $0 \leq s<a$, where $s=\sum_{i=1}^{n-1} h(i)$, $f:\{0,1, \ldots, n\} \times[0,+\infty)$ is continuous and $f(\cdot, u)$ is symmetric on $\{0,1, \ldots, n\}$ for all $u \geq 0$.

Remark 1 The conditions that $g$ and $h$ are symmetric on the different sets, which can guarantee the symmetry of associated kernel function for BVP (1.4)-(1.6). The kernel functions are then used to obtain the existence of symmetric positive solutions for BVP (1.4)(1.6) by constructing a suitable operator.

In order to study the existence of symmetric positive solutions of problem (1.4)-(1.6), we need the following lemmas.

Lemma 1.1 [16] Let $P$ be a cone of the real Banach space $E$ and $\Omega$ be a bounded open subset of $E$ and $\theta \in \Omega$. Assume $A: P \cap \bar{\Omega} \rightarrow P$ is a completely continuous operator and satisfies $A u=\mu u, u \in P \cap \partial \Omega, \mu<1$. Then $i(A, P \cap \Omega, P)=1$.

Lemma 1.2 [16] Suppose $A: P \cap \bar{\Omega} \rightarrow P$ is a completely continuous operator, and satisfies

(1) $\inf _{u \in P \cap \partial \Omega}\|A u\|>0$;

(2) $A u=\mu u, u \in P \cap \partial \Omega, \mu \notin(0,1]$.

Then $i(A, P \cap \Omega, P)=0$.

Lemma 1.3 (Hölder) Suppose $u=\left\{u_{1}, u_{2}, \ldots, u_{n}\right\}$ is a real-valued column, let

$$
\|u\|_{p}= \begin{cases}\left(\sum_{k=1}^{n}\left|u_{k}\right|^{p}\right)^{1 / p}, & 0<p<\infty, \\ \sup _{k \in\{1,2, \ldots, n\}}\left|u_{k}\right|, & p=\infty,\end{cases}
$$

where $p, q$ satisfy the condition $\frac{1}{p}+\frac{1}{q}=1$, which are called conjugate exponents, and $q=\infty$ for $p=1$. If $1 \leq p \leq \infty$, then

$$
\|u v\|_{1} \leq\|u\|_{p}\|v\|_{q}
$$


which can be denoted as

$$
\sum_{k=1}^{n}\left|u_{k} v_{k}\right| \leq \begin{cases}\left(\sum_{k=1}^{n}\left|u_{k}\right|^{p}\right)^{1 / p}\left(\sum_{k=1}^{n}\left|v_{k}\right|^{q}\right)^{1 / q}, & 1<p<\infty, \\ \left(\sum_{k=1}^{n}\left|u_{k}\right|\right)\left(\sup _{k \in\{1,2, \ldots, n\}}\left|v_{k}\right|\right), & p=1, \\ \left(\sup _{k \in\{1,2, \ldots, n\}}\left|u_{k}\right|\right)\left(\sum_{k=1}^{n}\left|v_{k}\right|\right), & p=\infty .\end{cases}
$$

\section{Preliminaries}

Let $E=\{u(k):\{0,1, \ldots, n\} \rightarrow \mathbb{R}\}$. It is well known that $E$ is a real Banach space with the norm $\|\cdot\|$ defined by $\|u\|=\max _{k \in\{0,1, \ldots, n\}}|u(k)|$. Let $K$ be a cone of $E$,

$$
K_{r}=\{u \in K:\|u\| \leq r\}, \quad \partial K_{r}=\{u \in K:\|u\|=r\},
$$

where $r>0$.

In our main results, we will use the following lemmas.

Lemma 2.1 Assume that $\left(\mathrm{A}_{1}\right)$ holds. Then for any $y \in E$, the BVP

$$
\begin{aligned}
& -\Delta(g(k-1) \Delta u(k-1))=y(k), \quad k \in\{1, \ldots, n-1\}, \\
& a u(0)-b g(0) \Delta u(0)=\sum_{i=1}^{n-1} h(i) u(i), \\
& a u(n)+b g(n-1) \Delta u(n-1)=\sum_{i=1}^{n-1} h(i) u(i)
\end{aligned}
$$

has a unique solution u given by

$$
u(k)=\sum_{i=1}^{n-1} H(k, i) y(i)
$$

where

$$
\begin{aligned}
& H(k, i)=G(k, i)+\frac{1}{a-s} \sum_{\tau=1}^{n-1} G(\tau, i) h(\tau), \\
& G(k, i)=\frac{1}{\Delta} \begin{cases}\left(b+a \sum_{j=k}^{n-1} \frac{1}{g(j)}\right)\left(b+a \sum_{j=0}^{i-1} \frac{1}{g(j)}\right), & 0 \leq i<k, \\
\left(b+a \sum_{j=i}^{n-1} \frac{1}{g(j)}\right)\left(b+a \sum_{j=0}^{k-1} \frac{1}{g(j)}\right), & k \leq i \leq n,\end{cases}
\end{aligned}
$$

and $\Delta=2 a b+a^{2} \sum_{j=0}^{n-1} \frac{1}{g(j)}, s=\sum_{i=1}^{n-1} h(i)$.

Proof From the properties of the difference operator, it is easy to see that

$$
-g(k) \Delta u(k)+g(k-1) \Delta u(k-1)=y(k),
$$

then we have

$$
-g(1) \Delta u(1)+g(0) \Delta u(0)=y(1),
$$




$$
\begin{aligned}
& -g(2) \Delta u(2)+g(1) \Delta u(1)=y(2), \\
& \ldots \\
& -g(k) \Delta u(k)+g(k-1) \Delta u(k-1)=y(k) .
\end{aligned}
$$

From the above equalities, we can obtain

$$
-g(k) \Delta u(k)+g(0) \Delta u(0)=\sum_{i=1}^{k} y(i)
$$

Let $g(0) \Delta u(0)=A$, then

$$
\Delta u(k)=\frac{1}{g(k)} A-\frac{1}{g(k)} \sum_{i=1}^{k} y(i)
$$

that is,

$$
u(k+1)-u(k)=\frac{1}{g(k)} A-\frac{1}{g(k)} \sum_{i=1}^{k} y(i) .
$$

So,

$$
\begin{aligned}
& u(1)-u(0)=\frac{1}{g(0)} A, \\
& u(2)-u(1)=\frac{1}{g(1)} A-\frac{1}{g(1)} \sum_{i=1}^{1} y(i), \\
& u(3)-u(2)=\frac{1}{g(2)} A-\frac{1}{g(2)} \sum_{i=1}^{2} y(i), \\
& \ldots \\
& u(k)-u(k-1)=\frac{1}{g(k-1)} A-\frac{1}{g(k-1)} \sum_{i=1}^{k-1} y(i) .
\end{aligned}
$$

It follows that

$$
u(k)=u(0)+A \sum_{j=0}^{k-1} \frac{1}{g(j)}-\sum_{j=1}^{k-1} \frac{1}{g(j)} \sum_{i=1}^{j} y(i) .
$$

By the boundary conditions, we get

$$
\begin{aligned}
& a u(0)-b A=\sum_{i=1}^{n-1} h(i) u(i), \\
& a u(0)+\left(b+a \sum_{j=0}^{n-1} \frac{1}{g(j)}\right) A=\sum_{i=1}^{n-1} h(i) u(i)+a \sum_{j=1}^{n-1} \frac{1}{g(j)} \sum_{i=1}^{j} y(i)+b \sum_{i=1}^{n-1} y(i) .
\end{aligned}
$$


Then

$$
\begin{aligned}
& A=\frac{1}{2 b+a \sum_{j=0}^{n-1} \frac{1}{g(j)}}\left(a \sum_{j=1}^{n-1} \frac{1}{g(j)} \sum_{i=1}^{j} y(i)+b \sum_{i=1}^{n-1} y(i)\right), \\
& u(0)=\frac{b}{2 a b+a^{2} \sum_{j=0}^{n-1} \frac{1}{g(j)}}\left(a \sum_{j=1}^{n-1} \frac{1}{g(j)} \sum_{i=1}^{j} y(i)+b \sum_{i=1}^{n-1} y(i)\right)+\frac{1}{a} \sum_{i=1}^{n-1} h(i) u(i) .
\end{aligned}
$$

Thus,

$$
\begin{aligned}
u(k)= & \frac{1}{a} \sum_{i=1}^{n-1} h(i) u(i)+\frac{b}{2 a b+a^{2} \sum_{j=0}^{n-1} \frac{1}{g(j)}}\left(a \sum_{j=1}^{n-1} \frac{1}{g(j)} \sum_{i=1}^{j} y(i)+b \sum_{i=1}^{n-1} y(i)\right) \\
& +\sum_{j=0}^{k-1} \frac{1}{g(j)} \cdot \frac{1}{2 b+a \sum_{j=0}^{n-1} \frac{1}{g(j)}}\left(a \sum_{j=1}^{n-1} \frac{1}{g(j)} \sum_{i=1}^{j} y(i)+b \sum_{i=1}^{n-1} y(i)\right) \\
& -\sum_{j=1}^{k-1} \frac{1}{g(j)} \sum_{i=1}^{j} y(i) \\
= & \frac{1}{a} \sum_{i=1}^{n-1} h(i) u(i)+\sum_{i=1}^{n-1} G(k, i) y(i),
\end{aligned}
$$

where $G(k, i)$ is defined by (2.5). Multiplying the above equation with $h(k)$, and summing from 1 to $n-1$, we can get

$$
\sum_{i=1}^{n-1} h(i) u(i)=\frac{a}{a-\sum_{k=2}^{n-1} h(k)} \sum_{k=1}^{n-1} h(k) \sum_{i=1}^{n-1} G(k, i) y(i) .
$$

One deduces that

$$
\begin{aligned}
u(k) & =\sum_{i=1}^{n-1} G(k, i) y(i)+\frac{1}{a-\sum_{k=1}^{n-1} h(k)} \sum_{k=1}^{n-1} h(k) \sum_{i=1}^{n-1} G(k, i) y(i) \\
& =\sum_{i=1}^{n-1} H(k, i) y(i),
\end{aligned}
$$

where $H(k, i)$ is defined by (2.4). The proof is complete.

From the above work, we can prove that $H(k, i)$ and $G(k, i)$ have the following properties.

Proposition 2.1 If $\left(\mathrm{A}_{1}\right)$ holds, then we have

$$
\begin{aligned}
& H(k, i)>0, \quad G(k, i)>0, \quad \text { for } k, i \in\{0,1, \ldots, n\} ; \\
& G(n-k, n-i)=G(k, i), \quad H(n-k, n-i)=H(k, i), \quad \text { for } k, i \in\{0,1, \ldots, n\} ; \\
& \frac{1}{\Delta} b^{2} \leq G(k, i) \leq G(i, i) \leq \frac{1}{\Delta} D, \quad \frac{1}{\Delta} a b^{2} \gamma \leq H(k, i) \leq H(i, i) \leq \frac{1}{\Delta} a \gamma D,
\end{aligned}
$$

where $D=\left(b+a \sum_{j=0}^{n} \frac{1}{g(j)}\right)^{2}, \gamma=\frac{1}{a-s}, k, i \in\{0,1, \ldots, n\}$. 
Proof It is clear that (2.6) holds. Now we prove (2.7) holds.

If $i \in\{0,1, \ldots, k-1\}$, then $n-i \geq n-k$, from (2.5) and $\left(\mathrm{A}_{1}\right)$ we get

$$
\begin{aligned}
G(n-k, n-i) & =\frac{1}{\Delta}\left(b+a \sum_{j=n-i}^{n-1} \frac{1}{g(j)}\right)\left(b+a \sum_{j=0}^{n-k-1} \frac{1}{g(j)}\right) \\
& =\frac{1}{\Delta}\left(b+a \sum_{j=n-i}^{n-1} \frac{1}{g(n-1-j)}\right)\left(b+a \sum_{j=0}^{n-k-1} \frac{1}{g(n-1-j)}\right) \\
& =\frac{1}{\Delta}\left(b+a \sum_{j=0}^{i-1} \frac{1}{g(j)}\right)\left(b+a \sum_{j=k}^{n-1} \frac{1}{g(j)}\right) \\
& =G(k, i), \quad i \in\{0,1, \ldots, k-1\} .
\end{aligned}
$$

Similarly, we can prove that $G(n-k, n-i)=G(k, i), i \in\{k, \ldots, n\}$. So we have $G(n-k, n-$ $i)=G(k, i)$, for $k, i \in\{0,1, \ldots, n\}$. From $(2.4)$ and $\left(\mathrm{A}_{1}\right)$, we have

$$
\begin{aligned}
H(n-k, n-i) & =G(n-k, n-i)+\frac{1}{a-s} \sum_{\tau=1}^{n-1} G(\tau, n-i) h(\tau) \\
& =G(k, i)+\frac{1}{a-s} \sum_{\tau=1}^{n-1} G(n-\tau, i) h(n-\tau) \\
& =G(k, i)+\frac{1}{a-s} \sum_{\tau=1}^{n-1} G(\tau, i) h(\tau) \\
& =H(k, i) .
\end{aligned}
$$

So, (2.7) is established. Next we prove (2.8) holds. In fact, for $k, i \in\{0,1, \ldots, n\}$, if $i \in$ $\{0,1, \ldots, k-1\}$, then

$$
\begin{aligned}
G(k, i) & =\frac{1}{\Delta}\left(b+a \sum_{j=k}^{n-1} \frac{1}{g(j)}\right)\left(b+a \sum_{j=0}^{i-1} \frac{1}{g(j)}\right) \\
& \leq \frac{1}{\Delta}\left(b+a \sum_{j=i}^{n-1} \frac{1}{g(j)}\right)\left(b+a \sum_{j=0}^{i-1} \frac{1}{g(j)}\right) \\
& =G(i, i) \\
& \leq \frac{1}{\Delta}\left(b+a \sum_{j=0}^{n} \frac{1}{g(j)}\right)\left(b+a \sum_{j=0}^{n} \frac{1}{g(j)}\right) \\
& \leq \frac{1}{\Delta}\left(b+a \sum_{j=0}^{n} \frac{1}{g(j)}\right)^{2} \\
& =\frac{1}{\Delta} D .
\end{aligned}
$$

Similarly, we can prove that $G(k, i) \leq G(i, i) \leq \frac{1}{\Delta} D$, for $i \in\{k, k+1, \ldots, n\}$. Therefore $G(k, i) \leq G(i, i) \leq \frac{1}{\Delta} D$. For $k, i \in\{0,1, \ldots, n\}$, we can get 


$$
\begin{aligned}
H(k, i) & =G(k, i)+\frac{1}{a-s} \sum_{\tau=1}^{n-1} G(\tau, i) h(\tau) \\
& \leq G(i, i)+\frac{1}{a-s} \sum_{\tau=1}^{n-1} G(\tau, i) h(\tau) \\
& =H(i, i) \\
& \leq G(i, i)+\frac{1}{a-s} \sum_{\tau=1}^{n-1} G(\tau, \tau) h(\tau) \\
& \leq \frac{1}{\Delta} D+\frac{1}{\Delta} D \frac{1}{a-s} \sum_{\tau=1}^{n-1} h(\tau) \\
& =\frac{1}{\Delta}\left(1+\frac{1}{a-s} \sum_{\tau=1}^{n-1} h(\tau)\right) D \\
& =\frac{a}{\Delta(a-s)} D=\frac{1}{\Delta} a \gamma D .
\end{aligned}
$$

On the other hand, from (2.5), we have

$$
G(k, i) \geq \frac{1}{\Delta}\left(b+a \sum_{j=n}^{n-1} \frac{1}{g(j)}\right)\left(b+a \sum_{j=0}^{-1} \frac{1}{g(j)}\right)=\frac{1}{\Delta} b^{2} .
$$

So, by (2.4), for $k, i \in\{0,1, \ldots, n\}$, we can obtain

$$
\begin{aligned}
H(k, i) & =G(k, i)+\frac{1}{a-s} \sum_{\tau=1}^{n-1} G(\tau, i) h(\tau) \\
& \geq \frac{1}{\Delta} b^{2}+\frac{b^{2}}{(a-s) \Delta} \sum_{\tau=1}^{n-1} h(\tau) \\
& \geq \frac{1}{\Delta} b^{2}\left(1+\frac{1}{a-s} \sum_{\tau=1}^{n-1} h(\tau)\right) \\
& =\frac{1}{\Delta} b^{2} \frac{a}{a-s}=\frac{1}{\Delta} b^{2} a \gamma .
\end{aligned}
$$

Thus,

$$
\begin{aligned}
& \frac{1}{\Delta} b^{2} \leq G(k, i) \leq G(i, i) \leq \frac{1}{\Delta} D, \\
& \frac{1}{\Delta} b^{2} a \gamma \leq H(k, i) \leq H(i, i) \leq \frac{1}{\Delta} D a \gamma .
\end{aligned}
$$

The proof is completed.

Remark 2 The symmetry of $g(k)$ on $\{0,1, \ldots, n-1\}$ can guarantee that $G(k, i)$ is symmetric for $k, i \in\{0,1, \ldots, n\}$, and the symmetry of $h(k)$ on $\{0,1, \ldots, n\}$ can guarantee that $H(k, i)$ is symmetric for $k, i \in\{0,1, \ldots, n\}$. 
Next, we can construct a cone in $E$ by

$$
\begin{aligned}
K= & \{u \in E: u \geq 0, u(k) \text { is symmetric on }\{0,1, \ldots, n\}, \Delta(g(k) \Delta u(k)) \leq 0, \\
& \left.k \in\{0,1, \ldots, n-2\}, \text { and } \min _{k \in\{0,1, \ldots, n\}} u(k) \geq \delta_{*}\|u\|\right\},
\end{aligned}
$$

where $\delta_{*}=\frac{1}{D} b^{2}$. Then we define an operator

$$
(T u)(k)=\sum_{i=1}^{n-1} H(k, i) w(i) f(i, u(i))
$$

It can be observed that $u$ is a solution of problem (1.4)-(1.6) if and only if $u$ is a fixed point of operator $T$.

We can get the following lemma from Lemma 2.1.

Lemma 2.2 Suppose $\left(\mathrm{A}_{1}\right)$ holds. If $u$ is a solution of the equation

$$
u(k)=\operatorname{Tu}(k)=\sum_{i=1}^{n-1} H(k, i) w(i) f(i, u(i))
$$

then $u$ is a solution of $B V P(1.4)-(1.6)$.

Lemma 2.3 Assume $\left(\mathrm{A}_{1}\right)$ holds. Then $T(K) \subset K$ and $T: K \rightarrow K$ is completely continuous.

Proof For $u \in K$, from (2.9), we obtain $\Delta(g(k-1) \Delta T u(k-1))=-w(k) f(k, u(k)) \leq 0$. By Proposition 2.1 , it is to see that $(T u)(k) \geq 0$, for $k \in\{0,1, \ldots, n\}$. Using the fact that $w, u$, $f(k, u)$ are symmetric on $\{0,1, \ldots, n\}$, we have

$$
\begin{aligned}
(T u)(n-k) & =\sum_{i=1}^{n-1} H(n-k, i) w(i) f(i, u(i)) \\
& =\sum_{i=1}^{n-1} H(k, n-i) w(n-i) f(n-i, u(n-i)) \\
& =\sum_{i=1}^{n-1} H(k, i) w(i) f(i, u(i)) \\
& =(T u)(k),
\end{aligned}
$$

then $T u$ is symmetric on $\{0,1, \ldots, n\}$ for $k \in\{0,1, \ldots, n\}$. And from (2.8) we can see

$$
(T u)(k)=\sum_{i=1}^{n-1} H(k, i) w(i) f(i, u(i)) \leq \frac{1}{\Delta} a \gamma D \sum_{i=1}^{n-1} w(i) f(i, u(i))
$$

Thus,

$$
\|T u\| \leq \frac{1}{\Delta} \operatorname{a\gamma D} \sum_{i=1}^{n-1} w(i) f(i, u(i)) .
$$


Similarly, by (2.8) we obtain

$$
\begin{aligned}
(T u)(k) & =\sum_{i=1}^{n-1} H(k, i) w(i) f(i, u(i)) \\
& \geq \frac{1}{\Delta} a b^{2} \gamma \sum_{i=1}^{n-1} w(i) f(i, u(i)) \\
& =\frac{1}{\Delta} a \delta_{*} D \gamma \sum_{i=1}^{n-1} w(i) f(i, u(i)) \\
& \geq \delta_{*}\|T u\| .
\end{aligned}
$$

Thus, $T u \in K$ and $T(K) \subset K$. It is clear that $T: K \rightarrow K$ is completely continuous.

Remark 3 The symmetry of the kernel function $H(k, i)$ for $k, i \in\{0,1, \ldots, n\}$ can guarantee that $T u$ is symmetric on $\{0,1, \ldots, n\}$ for $u \in K$.

\section{Main results}

In this section, we will establish that problem (1.4)-(1.6) has at least one positive solution with Lemma 1.1 and Lemma 1.2. We need consider the following situations: $p>1, p=1$, $p=\infty$. Next, we will prove a theorem for $p>1$. At first, we define

$$
\|H\|=\sup _{i \in\{1,2, \ldots, n-1\}}|H(i, i)|, \quad\|H\|_{p}=\left(\sum_{i=1}^{n-1}|H(i, i)|^{p}\right)^{1 / p} .
$$

Let

$$
F^{\beta}=\lim _{u \rightarrow \beta} \sup \max _{k \in\{0,1, \ldots, n\}} \frac{f(k, u)}{u}, \quad F_{\beta}=\liminf _{u \rightarrow \beta} \min _{k \in\{0,1, \ldots, n\}} \frac{f(k, u)}{u},
$$

where $\beta$ denotes 0 or $\infty$, and

$$
\begin{aligned}
N^{-1}= & \max \left\{\|H\|_{p}\left(\sum_{i=1}^{n-1}|w(i)|^{q}\right)^{1 / q},\right. \\
& \left.\left(\sum_{i=1}^{n-1}|H(i, i)|\right)\left(\sup _{i \in\{1,2, \ldots, n-1\}}|w(i)|\right),\|H\|\left(\sum_{i=1}^{n-1}|w(i)|\right)\right\}, \\
L^{-1}= & \frac{1}{\Delta} \delta_{*} a \gamma m b^{2} .
\end{aligned}
$$

Theorem 3.1 Assume that conditions $\left(\mathrm{A}_{1}\right)$ hold. In addition, suppose that

$\left(\mathrm{A}_{2}\right) \quad 0<F^{0}<N$, and $L<F_{\infty}<\infty$, or

$\left(\mathrm{A}_{3}\right) 0<F^{\infty}<N$, and $L<F_{0}<\infty$

are satisfied. Then problem (1.4)-(1.6) has at least one symmetric positive solution.

Proof We only consider $\left(\mathrm{A}_{2}\right)$ case, $\left(\mathrm{A}_{3}\right)$ is similar to $\left(\mathrm{A}_{2}\right)$. If $0<F^{0}<N$, then there exist $r>0, \varepsilon_{0}>0$ such that $N-\varepsilon_{0}>0$ and for all $0<u \leq r$, we have

$$
f(k, u) \leq\left(N-\varepsilon_{0}\right) u \leq\left(N-\varepsilon_{0}\right) r, \quad k \in\{0,1, \ldots, n\} .
$$


For all $u \in \partial K_{r}$, from Lemma 1.3 we obtain

$$
\begin{aligned}
(T u)(k) & =\sum_{i=1}^{n-1} H(k, i) w(i) f(i, u(i)) \\
& \leq \sum_{i=1}^{n-1} H(k, i) w(i)\left(N-\varepsilon_{0}\right) r \\
& \leq \sum_{i=1}^{n-1} H(i, i) w(i)\left(N-\varepsilon_{0}\right) r \\
& \leq\left(\sum_{i=1}^{n-1}|H(i, i)|^{p}\right)^{1 / p}\left(\sum_{i=1}^{n-1}|w(i)|^{q}\right)^{1 / q}\left(N-\varepsilon_{0}\right) r \\
& \leq N^{-1}\left(N-\varepsilon_{0}\right) r \\
& \leq r .
\end{aligned}
$$

So $T u \neq \lambda u$, for $\forall u \in \partial K_{r}, \lambda \geq 1$. From Lemma 1.1, we can get $i\left(T, K_{r}, K\right)=1$. Next, we prove it satisfies Lemma 1.2. Because $L<F_{\infty}<\infty$, there exist $R>\delta_{*} r>0, \varepsilon_{1}>0$ such that

$$
f(k, u) \geq\left(L+\varepsilon_{1}\right) u, \quad \forall u \geq R, k \in\{0,1, \ldots, n\} .
$$

Let $r^{*}=\delta_{*}^{-1} R$, then $r^{*}>r$, and

$$
\min _{k \in\{0,1, \ldots, n\}} u(k) \geq \delta_{*}\|u\|=R, \quad \forall u \in \partial K_{r} .
$$

Now we prove that $T u \neq \lambda u, \forall u \in \partial K_{r}, 0<\lambda \leq 1$. If not, then there exist $u_{0} \in \partial K_{r^{*}}$ and $0<\lambda_{0} \leq 1$ such that $T u_{0}=\lambda_{0} u_{0}$; thus we have

$$
\begin{aligned}
r^{*} & \geq u_{0}(k)=\lambda_{0}^{-1}\left(T u_{0}\right)(k) \\
& =\lambda_{0}^{-1} \sum_{i=1}^{n-1} H(k, i) w(i) f(i, u(i)) \\
& \geq \frac{1}{\Delta} a b^{2} \gamma\left(L+\varepsilon_{1}\right) \sum_{i=1}^{n-1} w(i) u(i) \\
& \geq \frac{1}{\Delta} a b^{2} \gamma\left(L+\varepsilon_{1}\right) R \sum_{i=1}^{n-1} w(i) \\
& =\frac{1}{\Delta} a b^{2} \gamma\left(L+\varepsilon_{1}\right) \delta_{*} r^{*} \sum_{i=1}^{n-1} w(i) \\
& \geq \frac{1}{\Delta} a b^{2} \gamma\left(L+\varepsilon_{1}\right) \delta_{*} r^{*} m \\
& =L^{-1}\left(L+\varepsilon_{1}\right) r^{*} \\
& =r^{*}\left(1+\frac{\varepsilon_{1}}{L}\right)>r^{*},
\end{aligned}
$$


i.e., $r^{*}>r^{*}$, which is a contradiction. In addition, because $(T u)(k) \geq r^{*}\left(1+\frac{\varepsilon_{1}}{L}\right)>r^{*}$, so $\inf _{u \in \partial K_{r^{*}}}\|T u\| \geq r^{*}>0$, from Lemma 1.2 we have $i\left(T, K_{r^{*}}, K\right)=0$. On the other hand, from the above work with the additivity of the fixed point index, we get

$$
i\left(T, K_{r^{*}}-\overline{K_{r}}, K\right)=i\left(T, K_{r^{*}}, K\right)-i\left(T, K_{r}, K\right)=0-1=-1 .
$$

So, $T$ has at least one fixed point $u^{*}$ on $K_{r^{*}}-\overline{K_{r}}$. Then it follows that problem (1.4)-(1.6) has a symmetric positive solution $u^{*}$. The proof is complete.

Remark 4 From the proof of Theorem 3.1, we can establish that problem (1.4)-(1.6) has another nonnegative solution $u^{* *}, u^{* *} \in K_{r}$.

The following corollary deals with the case $p=1$.

Corollary 3.1 Suppose that $\left(\mathrm{A}_{1}\right),\left(\mathrm{A}_{2}\right)$ hold. Then problem (1.4)-(1.6) has at least one symmetric positive solution.

Proof It is similar to the proof of Theorem 3.1. Let $\left(\sum_{i=1}^{n-1}|H(i, i)|\right)\left(\sup _{i \in\{1, \ldots, n-1\}}|w(i)|\right)$ replace $\left(\sum_{i=1}^{n-1}|H(i, i)|^{p}\right)^{1 / p}\left(\sum_{i=1}^{n-1}|w(i)|^{q}\right)^{1 / q}$ and repeat the argument of Theorem 3.1.

Finally, we consider the case of $p=\infty$.

Corollary 3.2 Assume that $\left(\mathrm{A}_{1}\right),\left(\mathrm{A}_{2}\right)$ hold. Then problem (1.4)-(1.6) has at least one symmetric positive solution.

Proof It is similar to the proof of Theorem 3.1. For all $u \in \partial K_{r}$, we have

$$
\begin{aligned}
(T u)(k) & =\sum_{i=1}^{n-1} H(k, i) w(i) f(i, u(i)) \\
& \leq \sum_{i=1}^{n-1} H(i, i) w(i)\left(N-\varepsilon_{0}\right) r \\
& \leq\left(\sup _{i \in\{1,2, \ldots, n-1\}}|H(i, i)|\right)\left(\sum_{i=1}^{n-1}|w(i)|\right)\left(N-\varepsilon_{0}\right) r \\
& \leq N^{-1}\left(N-\varepsilon_{0}\right) r \\
& <r .
\end{aligned}
$$

So $T u \neq \lambda u, u \in \partial K_{r}, \lambda \geq 1$. By Lemma 1.1, we can get $i\left(T, K_{r}, K\right)=1$. This together with $i\left(T, K_{r^{*}}, K\right)=0$ in the proof of Theorem 3.1 completes the proof.

The authors declare that they have no competing interests. 


\section{Author details}

'School of Electrical Engineering, Hebei University of Science and Technology, Shijiazhuang, Hebei 050018, P.R. China.

${ }^{2}$ College of Sciences, Hebei University of Science and Technology, Shijiazhuang, Hebei 050018, P.R. China.

\section{Acknowledgements}

The authors express their sincere thanks to the referees for the careful and details reading of the manuscript and very helpful suggestions. The project was supported by the Natural Science Foundation of China (11371120), the Natural Science Foundation of Hebei Province (A2013208147) and the Education Department of Hebei Province Science and Technology Research Project (Z2014095).

\section{Received: 23 March 2014 Accepted: 25 August 2014 Published: 09 Sep 2014}

\section{References}

1. Cannon, JR: The solution of the heat equation subject to the specification of energy. Q. Appl. Math. 21, 155-160 (1963)

2. Ionkin, NI: Solution of boundary value problem in heat conduction theory with nonlocal boundary conditions. Differ. Equ. 13, 294-304 (1977)

3. Chegis, RY: Numerical solution of heat conduction problem with an integral boundary condition. Liet. Mat. Rink. 24, 209-215 (1984)

4. Boucherif, A: Second-order boundary value problems with integral boundary conditions. Nonlinear Anal. 70, 364-371 (2009)

5. Infante, G: Eigenvalues and positive solutions of ODEs involving integral boundary conditions. Discrete Contin. Dyn. Syst. suppl., 436-442 (2005)

6. Yang, Z: Positive solutions of a second order integral boundary value problem. J. Math. Anal. Appl. 321, 751-765 (2006)

7. Ahmad, B, Alsaedi, A, Alghamdi, BS: Analytic approximation of solutions of the forced Duffing equation with integral boundary conditions. Nonlinear Anal., Real World Appl. 9, 1727-1740 (2008)

8. Ahmad, B, Alsaedi, A: Existence of approximate solutions of the forced Duffing equation with discontinuous type integral boundary conditions. Nonlinear Anal., Real World Appl. 10, 358-367 (2009)

9. Feng, M, Zhang, $X, G$, W: New existence results for higher-order nonlinear fractional differential equation with integral boundary conditions. Bound. Value Probl. 2011, 720702 (2011)

10. Feng, M: Existence of symmetric positive solutions for a boundary value problem with integral boundary conditions. Appl. Math. Lett. 24, 1419-1427 (2011)

11. Csavinszky, P: Universal approximate solution of the Thomas-Fermi equation for ions. Phys. Rev. A 8, $1688-1701$ (1973)

12. Granas, A, Guenther, RB, Lee, JW: Nonlinear boundary value problems for ordinary differential equations. Diss. Math. 244, 128 pp. (1985)

13. Granas, A, Guenther, RB, Lee, JW: A note on the Thomas-Fermi equations. Z. Angew. Math. Mech. 61, $240-241$ (1981)

14. Luning, CD, Perry, WL: Positive solutions of negative exponent generalized Emden-Fowler boundary value problems. SIAM J. Math. Anal. 12,874-879 (1981)

15. Wong, JSW: On the generalized Emden-Fowler equations. SIAM Rev. 17, 339-360 (1975)

16. Guo, D, Lakshmikantham, V: Nonlinear Problems in Abstract Cones. Academic Press, New York (1988)

10.1186/1687-1847-2014-237

Cite this article as: Guo et al.: The existence of symmetric positive solutions for a seconder-order difference equation with sum form boundary conditions. Advances in Difference Equations 2014, 2014:237

\section{Submit your manuscript to a SpringerOpen ${ }^{\circ}$ journal and benefit from:}

- Convenient online submission

- Rigorous peer review

- Immediate publication on acceptance

Open access: articles freely available online

- High visibility within the field

- Retaining the copyright to your article 